\title{
SPECIALIZATION OF FORMS IN THE PRESENCE OF CHARACTERISTIC 2: FIRST STEPS
}

\author{
MANFRED KNEBUSCH \\ UNIVERSITY OF REGENSBURG
}

\section{INTRODUCTION}

We outline a specialization theory of quadratic and (symmetric) bilinear forms with respect to a place $\lambda: K \rightarrow L \cup \infty$. Here $K, L$ denote fields of any characteristic. We have to make a distinction between bilinear forms and quadratic forms and study them both over fields and valuation rings.

For bilinear forms this turns out to be essentially as easy as in the case char $L \neq 2$, albeit no general cancellation law holds for nondegenerate bilinear forms over a valuation domain $\mathcal{O}$, in which 2 is not a unit. For quadratic forms things are more difficult mainly for two reasons. 1) Forms cannot be diagonalized. 2) The quasilinear part of an anisotropic form over $\mathcal{O}$ may become isotropic over the residue class field of $\mathcal{O}$.

Nevertheless a somewhat restricted specialization theory for quadratic forms is possible which is good enough to establish a fully fledged generic splitting theory. On the other hand it seems, that for bilinear forms no generic splitting is possible. (Most probably there does not exist a "generic zero field" for a bilinear form over a field of characteristic 2.) But specialization of bilinear forms is nevertheless important for generic splitting of quadratic forms, since a bilinear form and a quadratic form can be multiplied via tensor product to give another quadratic form.

All this is explicated in a recent book by the author [Spez]. The book contains more material than outlined here. In particular its last chapter IV gives a specialization theory of forms under "quadratic places", much more tricky than the theory for ordinary places. Miraculously this leads to a generic splitting theory with respect to quadratic places which is as satisfactory as for ordinary places.

If $\varphi$ is a quadratic form over a field $K$ which has "good reduction" with respect to a place $\lambda: K \rightarrow L \cup \infty$ then our specialization theory gives a quadratic form $\lambda_{*}(\varphi)$ over $L$. We also develop a theory of "weak specialization", which associates to $\varphi$ only a Witt class $\lambda_{W}(\varphi)$ of forms over $L$, but under a more general condition on $\varphi$ than just having good reduction. In the present article weak specialization plays only an auxiliary role in order to define specializations $\lambda_{*}(\varphi)$. But weak specialization is a key notion in establishing the specialization theory for quadratic places (not described here, cf. [Spez, Chap. IV]). 
The book [Spez] is in German. It is now in the process of translation into English by Thomas Unger. A preprint of the first two chapters is available [Spez'].

Everything said in $\S 2-\S 6$ of the present article can be found with proofs and/or references in these two chapters. I have freely borrowed from passages in Unger's translation. I also give almost no references here to the work of others, referring to the references in the book instead.

I thank Professors Ricardo Baeza and Ulf Rehmann for help in preparing this manuscript.

\section{SPECIALIZATION OF SYMMETRIC BILINEAR FORMS}

We are given a place $\lambda: K \rightarrow L \cup \infty$ and a symmetric bilinear form $\varphi$, i.e., a polynomial

$$
\varphi(x, y)=\sum_{i, j=1}^{n} a_{i j} x_{i} y_{i}
$$

over $K$ in two sets of variables $x=\left(x_{1}, \ldots, x_{n}\right), y=\left(y_{1}, \ldots, y_{n}\right)$, with coefficients $a_{i j}=a_{j i} \in K$. Under suitable conditions ("good reduction", see below) we want to associate to $\varphi$ a symmetric bilinear form $\lambda_{*}(\varphi)$ over $L$ in a reasonable way.

We assume that $\varphi$ is nondegenerate, i.e., $\operatorname{det}\left(a_{i j}\right) \in K^{*}$, and we want that $\lambda_{*}(\varphi)$ is again a nondegenerate form, of the same dimension $n=\operatorname{dim} \varphi$ as $\varphi$.

For the rest of this section a form always means a nondegenerate symmetric bilinear form. We denote the form $\varphi$ above by the symmetric matrix $\left(a_{i j}\right)$. Nondegeneracy of $\varphi$ means that $\operatorname{det}\left(a_{i j}\right) \neq 0$.

We call two forms $\varphi=\left(a_{i j}\right), \psi=\left(b_{i j}\right)$ isometric ( $=$ isomorphic), and write $\varphi \cong \psi$, if $\operatorname{dim} \varphi=\operatorname{dim} \psi$ and $\psi$ is obtained from $\varphi$ by a linear change of coordinates, in matrix notation

$$
\left(b_{i j}\right)={ }^{t} U\left(a_{i j}\right) U
$$

with some $U \in G L(n, K)$.

Let $\mathcal{O}_{\lambda}$ denote the valuation ring of $\lambda, \mathcal{O}_{\lambda}=\{x \in K \mid \lambda(x) \neq \infty\}$.

Definition 2.1. We say that the form $\varphi=\left(a_{i j}\right)$ has good reduction with respect to the place $\lambda: K \rightarrow L \cup \infty$, if there exists a symmetric matrix $\left(b_{i j}\right)$ with coefficients in $\mathcal{O}_{\lambda}$ and $\operatorname{det}\left(b_{i j}\right)$ a unit of $\mathcal{O}_{\lambda}$, such that $\varphi$ is isometric to the form $\left(b_{i j}\right)$ over $K$. Alternatively we then say that $\varphi$ is $\lambda$-unimodular, and we call an isometry $\varphi \cong\left(b_{i j}\right)$ a $\lambda$-unimodular representation of $\varphi$.

In this situation we are tempted to define

$$
\lambda_{*}(\varphi):=\left(\lambda\left(b_{i j}\right)\right),
$$

hoping that - up to isometry - the form $\left(\lambda\left(b_{i j}\right)\right)$ does not depend on the choice of the $\lambda$-unimodular representation of $\varphi$.

(N.B.: We do not care to identify a form with an isometric form, thus abusively speaking of "forms" instead of isometry classes of forms.).

In this hope justified? The answer will be

"Yes", if char $L \neq 2$, and "Nearly", if $\operatorname{char} L=2$.

Our approach to the question will be via Witt rings. We briefly recall the definition of the Witt ring $\mathrm{W}(K)$. We call two forms $\varphi$ and $\psi$ over $K$ stably isometric, if there exists a form $\chi$ over $K$ such that $\varphi \perp \chi \cong \psi \perp \chi$. We then write 
$\varphi \approx \psi$. If $\operatorname{char} K \neq 2$ then $\varphi \approx \psi$ implies $\varphi \cong \psi$ by Witt's cancellation theorem. For char $K=2$, this is false.

Definition 2.2. We say that two forms $\varphi$ and $\psi$ over $K$ are Witt equivalent, and then write $\varphi \sim \psi$, if there exist numbers $r, s \in \mathbb{N}_{0}$ such that

$$
\varphi \perp r \times\left(\begin{array}{cc}
0 & 1 \\
1 & 0
\end{array}\right) \approx \psi \perp s \times\left(\begin{array}{cc}
0 & 1 \\
1 & 0
\end{array}\right) .
$$

The Witt (equivalence) class of a form $\varphi$ will be denoted by $\{\varphi\}$.

Witt classes can be added and multiplied as follows:

$$
\begin{aligned}
\{\varphi\}+\{\psi\} & :=\{\varphi \perp \psi\}, \\
\{\varphi\} \cdot\{\psi\} & :=\{\varphi \otimes \psi\},
\end{aligned}
$$

where $\perp$ and $\otimes$ denote the usual orthogonal sum and tensor product of symmetric bilinear forms. In this way the set of the Witt classes over $K$ becomes a well defined commutative ring with 1 , the Witt ring $\mathrm{W}(K)$. The zero element is given by the class $\left\{\left(\begin{array}{ll}0 & 1 \\ 1 & 0\end{array}\right)\right\}$ (or by the zero-dimensional form $\varphi=0$, which we admit), and the unit element by the class $\{(1)\}$ of the one-dimensional form (1). For any form $\varphi$, we have $\{\varphi\}+\{-\varphi\}=0$.

A good insight into Witt equivalence is given by the following Proposition 2.3. First a bit of notation. A form $\varphi$ of dimension $n$ is called isotropic, if there exists some $x \in K^{n}, x \neq 0$, with $\varphi(x, x)=0$, and anisotropic otherwise. $\varphi$ is called metabolic if

$$
\varphi \cong\left(\begin{array}{cc}
a_{1} & 1 \\
1 & 0
\end{array}\right) \perp \ldots \perp\left(\begin{array}{cc}
a_{r} & 1 \\
1 & 0
\end{array}\right)
$$

for some $i>0$ and $a_{1}, \ldots, a_{r} \in K$.

Every form $\varphi$ has a decomposition

$$
\varphi \cong \varphi_{0} \perp \varphi_{1}
$$

with $\varphi_{0}$ anisotropic and $\varphi_{1}$ metabolic, called a Witt decomposition of $\varphi$.

Proposition 2.3. Let $\varphi \cong \varphi_{0} \perp \varphi_{1}$ and $\psi \cong \psi_{0} \perp \psi_{1}$ be Witt decompositions of two forms $\varphi$ and $\psi$. Then $\varphi \sim \psi$ iff $\varphi_{0} \cong \psi_{0}$.

In particular, the anisotropic part $\varphi_{0}$ of $\varphi$ is uniquely determined by $\varphi$ up to isometry. We call $\varphi_{0}$ the kernel form of $\varphi$ and write $\varphi_{0}=\operatorname{ker}(\varphi)$.

(Alternatively we may call $\varphi_{0}$ the anisotropic part of $\varphi$ and write $\varphi_{0}=\varphi_{a n}$.)

As a consequence of Proposition 2.3 we state

Corollary 2.4. $\varphi \approx \psi$ iff $\varphi \sim \psi$ and $\operatorname{dim} \varphi=\operatorname{dim} \psi$.

Given elements $a_{1}, \ldots, a_{n} \in K^{*}$, we denote the diagonal form

$$
\left(\begin{array}{ccc}
a_{1} & & 0 \\
& \ddots & \\
0 & & a_{n}
\end{array}\right)
$$


more succinctly by $\left\langle a_{1}, \ldots, a_{n}\right\rangle$. We have the rules

$$
\begin{aligned}
\left\langle a_{1}, \ldots, a_{n}\right\rangle \perp\left\langle b_{1}, \ldots, b_{m}\right\rangle & \cong\left\langle a_{1}, \ldots, a_{n}, b_{1}, \ldots, b_{m}\right\rangle, \\
\left\langle a_{1}, \ldots, a_{n}\right\rangle \otimes\left\langle b_{1}, \ldots, b_{m}\right\rangle & \cong\left\langle a_{1} b_{1}, \ldots, a_{1} b_{m}, a_{2} b_{1}, \ldots, a_{n} b_{m}\right\rangle, \\
\langle a,-a\rangle & \cong\left(\begin{array}{cc}
0 & 1 \\
1 & 0
\end{array}\right)
\end{aligned}
$$

If for a form $\varphi$ there exists at least one vector $x$ with $\varphi(x, x) \neq 0$, then $\varphi$ has an orthogonal basis, i.e. $\varphi$ can be diagonalized, $\varphi \cong\left\langle a_{1}, \ldots, a_{n}\right\rangle$ for some $a_{i} \in K^{*}$. Otherwise $\varphi$ is an orthogonal sum $m \times\left(\begin{array}{ll}0 & 1 \\ 1 & 0\end{array}\right)$ of copies of the form $\left(\begin{array}{ll}0 & 1 \\ 1 & 0\end{array}\right)$, hence $\varphi \sim 0$. Thus the Witt ring $\mathrm{W}(K)$ is additively generated by the classes $\{\langle a\rangle\}$ with $a$ running through $K^{*}$.

As a very special case of Proposition 1.1 we observe that two classes $\{\langle a\rangle\},\{\langle b\rangle\}$ are equal iff $\langle a\rangle \cong\langle b\rangle$ iff the square classes $a K^{*}$ and $b K^{* 2}$ are equal. In the following we identify the set of these Witt classes, and also the set of isometry classes of onedimensional forms over $K$, with the group $Q(K)=K^{*} / K^{* 2}$ of square classes of $K$.

We have $\langle a\rangle \otimes\langle b\rangle=\langle a b\rangle$, and thus may - and will - regard $Q(K)$ as a subgroup of the group $\mathrm{W}(K)^{*}$ of units of the Witt ring $\mathrm{W}(K)$.

We return to the place $\lambda: K \rightarrow L \cup \infty$ with valuation ring $\mathcal{O}:=\mathcal{O}_{\lambda}$. Our specialization theory of bilinear forms is based on the following theorem.

Theorem 2.5. There exists a well defined additive map $\lambda_{W}: \mathrm{W}(K) \rightarrow \mathrm{W}(L)$, which can be characterized as follows. If $a$ is a unit of $\mathcal{O}$, then $\lambda_{W}(\langle a\rangle)=\langle\lambda(a)\rangle$. If a square class $\langle a\rangle=a K^{* 2}$ does not contain a unit of $\mathcal{O}$, then $\lambda_{W}(\langle a\rangle)=0 .{ }^{1}$

This can be proved by using a description of the additive group of $\mathrm{W}(K)$ by generators and relations. We have an additive map $\Lambda_{W}$ from the group ring $\mathbb{Z}[Q(K)]$ to $\mathrm{W}(L)$, which maps a group element $\langle a\rangle \in Q(K)$ to $\langle\lambda(a)\rangle$ if $a \in \mathcal{O}^{*}$, and to 0 if $a K^{* 2}$ does not contain a unit of $\mathcal{O}$.

The obvious surjection $\mathbb{Z}[Q(K)] \rightarrow \mathrm{W}(K)$ has a kernel $\mathfrak{a}$ which can be described explicitly (cf. [Spez, §2]).

One then verifies that $\Lambda_{W}(\mathfrak{a})=0$. Thus $\Lambda_{W}$ factors through an additive map $\lambda_{W}: \mathrm{W}(K) \rightarrow \mathrm{W}(L)$ with the properties stated in the theorem.

Proposition 2.6. Assume that the form $\varphi$ has good reduction under $\lambda$, and $\varphi \cong$ $\left(b_{i j}\right)$ is a $\lambda$-unimodular representation of $\varphi$. Then

$$
\lambda_{W}(\{\varphi\})=\left\{\left(\lambda\left(b_{i j}\right)\right)\right\}
$$

This is obvious from Theorem 1.3 if $\left(b_{i j}\right)$ is a diagonal matrix. In the general case one has to argue that the symmetric matrix can be "diagonalized over $\mathcal{O}$ ", i.e.,

\footnotetext{
${ }^{1}$ The letter $W$ in the notation $\lambda_{W}$ refers to "Witt" or "weak" (cf. also $§ 5$ ).
} 
there exists an equation

$$
\left(b_{i j}\right)={ }^{t} U\left(\begin{array}{ccc}
c_{1} & & 0 \\
& \ddots & \\
0 & & c_{n}
\end{array}\right) U
$$

with $c_{i} \in \mathcal{O}^{*}$ and $U \in G L(n, \mathcal{O})$.

This is not always true, but becomes true if we replace $\left(b_{i j}\right)$, say, by $\left(b_{i j}\right) \perp$ $\langle 1,-1\rangle$. The proof is best understood in the geometric setting to be developed in $\S 3$.

Corollary 2.7. Assume that $\varphi$ and $\psi$ are forms over $K$ with good reduction and that $\varphi \cong\left(a_{i j}\right), \psi \cong\left(b_{i j}\right)$ are $\lambda$-unimodular representations. If $\varphi \approx \psi$, then $\left(\lambda\left(b_{i j}\right)\right) \approx\left(\lambda\left(c_{i j}\right)\right)$.

Proof. We conclude by Theorem 2.5 that the forms $\left(\lambda\left(b_{i j}\right)\right)$ and $\left(\lambda\left(c_{i j}\right)\right)$ are Witt equivalent, and then by Proposition 2.4 that they are stably isometric, since they have the same dimension.

In particular, if $\varphi \cong\left(a_{i j}\right)$ and $\varphi \cong\left(b_{i j}\right)$ are two $\lambda$-unimodular representations of a form $\varphi$ over $K$ then the forms $\left(\lambda\left(a_{i j}\right)\right)$ and $\left(\lambda\left(b_{i j}\right)\right)$ over $L$ are stably isometric. Abusively we call $\left(\lambda\left(a_{i j}\right)\right)$ "the" specialization of $\varphi$ under $\lambda$, and denote this form by $\lambda_{*}(\varphi)$, although $\lambda_{*}(\varphi)$ is uniquely determined by $\varphi$ and $\lambda$ only up to stable isometry.

\section{Bilinear Modules}

We now switch to the "geometric language" for bilinear and - later ( $\S 4)$ - quadratic forms. Everything said in this section is very well known.

We first fix the basic notation valid for the rest of the paper. $\mathcal{O}$ always denotes a valuation domain, $\mathfrak{m}$ its maximal ideal, $k=\mathcal{O} / \mathfrak{m}$ its residue class field and $K=$ $\operatorname{Quot}(\mathcal{O})$ its quotient field, $\mathcal{O}^{*}$ denotes the group of units of $\mathcal{O}$, hence $\mathcal{O}^{*}=\mathcal{O} \backslash$ m.

The case $\mathfrak{m}=\{0\}$, i.e., $\mathcal{O}=K$, is by no means excluded.

A bilinear module $M=(M, B)$ over $\mathcal{O}$ consists of an $\mathcal{O}$-module $M$ and a symmetric bilinear form $B: M \times M \rightarrow \mathcal{O}$. If nothing else is said, we tacitly assume that the $\mathcal{O}$-module $M$ is free of finite rank $n$. We write $n=\operatorname{dim} M$. If $e_{1}, \ldots, e_{n}$ is a basis of $M$, then $B$ is given by the symmetric $n \times n$-matrix $\left(a_{i j}\right)$ with $a_{i j}=B\left(e_{i}, e_{j}\right)$.

Abusively we denote $M=(M, B)$, or better, its isometry class by this matrix $\left(a_{i j}\right)$. If $e_{1}, \ldots, e_{n}$ is an orthogonal basis, $a_{i j}=a_{i} \delta_{i j}$, we denote the bilinear module $M$ also by $\left\langle a_{1}, \ldots, a_{n}\right\rangle$.

We call the bilinear module $M$ (or the form $B$ ) non degenerate if $B$ gives an isomorphism of $\mathcal{O}$-modules $x \mapsto B(x,-)$ from $M$ to its dual module $\check{M}=$ $\operatorname{Hom}_{\mathcal{O}}(M, \mathcal{O})$. This means that $\operatorname{det}\left(a_{i j}\right) \in \mathcal{O}^{*}$. We then also say that $M$ is a bilinear space over $\mathcal{O}$.

It is well known that if $M$ is a bilinear space containing a vector $x$ with $B(x, x) \in$ $\mathcal{O}^{*}$, then $M$ has an orthogonal basis, hence $M \cong\left\langle a_{1}, \ldots, a_{n}\right\rangle$ with $a_{i} \in \mathcal{O}^{*}$. This fills the gap in our sketch of proof of $\S 2$, Proposition 2.6.

We call a submodule $N$ of a bilinear space $M$ a subspace of $M$, if $N$ is a direct summand of the module $M$. It will be helpful to remember that every finitely 
generated torsion free $\mathcal{O}$-module is free. Thus a submodule $N$ of $M$ is a subspace iff $M / N$ is torsion free.

For any subset $S$ of a bilinear module $M$ the module

$$
S^{\perp}=\{x \in M \mid B(x, S)=0\}
$$

is a direct summand of $M$, since $M / S^{\perp}$ is clearly torsion free and finitely generated.

We call a bilinear space $M$ isotropic, if $M$ contains a subspace $U \neq 0$, which is "totally isotropic", i.e., $B(U, U)=0$, in other terms, $U \subset U^{\perp}$. Otherwise we call $M$ anisotropic.

Since $\mathcal{O}$ has no zero divisors, and every finitely generated ideal of $\mathcal{O}$ is principal, it is easily seen that $M$ is isotropic iff there exists a vector $x \neq 0$ in $M$ with $B(x, x)=0$.

Indeed, we may always write $x=c z$ with $c \in \mathcal{O}$ and $z$ a primitive vector of $M$, i.e., a vector $z$, such that $\mathcal{O} z$ is a direct summand of the module $M$. \{ N.B.: If $e_{1}, \ldots, e_{n}$ is a basis of $M$ and $z=a_{1} e_{1}+\ldots+a_{n} e_{n}$, then $z$ is primitive iff $a_{1} \mathcal{O}+\ldots+a_{n} \mathcal{O}=\mathcal{O}$. $\}$

We call $M$ metabolic if $M$ contains a subspace $U=U^{\perp}$. Equivalently we can say, that $M$ is metabolic iff $M$ contains a totally isotropic subspace $U$ with $2 \operatorname{dim} U=$ $\operatorname{dim} M$. Every metabolic space $M$ has an orthogonal decomposition

$$
M \cong\left(\begin{array}{cc}
a_{1} & 1 \\
1 & 0
\end{array}\right) \perp \ldots \perp\left(\begin{array}{cc}
a_{m} & 1 \\
1 & 0
\end{array}\right)
$$

with some $a_{i} \in \mathcal{O}$. Notice that in the case $\mathcal{O}=K$ our present terminology is in complete harmony with $\S 2$, identifying isometry classes of spaces and isometry classes of forms in the obvious way.

Every bilinear space $M$ is an orthogonal sum of an anisotropic space $M_{0}$ and a metabolic space $M_{1}$. But now, in contrast to the case $\mathcal{O}=K$ (cf. Prop. 2.3), the isometry class of $M_{0}$ usually is not uniquely determined by $M$, if $2 \notin \mathcal{O}^{*}$.

For the rest of this section "space" always means "bilinear space". Exactly as in $\S 2$ we define stable isometry $(\approx)$ and Witt equivalence $(\sim)$ of forms over $\mathcal{O}$, and then proceed as there to the Witt ring $\mathrm{W}(\mathcal{O})$ consisting of the Witt (equivalence) classes of spaces.

We denote the Witt class of a space $M$ by $\{M\}$. It turns out that $\{M\}=0$, i.e., $M \sim 0$, iff $M$ is metabolic. Also, for every space $M=(M, B)$ the space $(M, B) \perp(M,-B)$ is metabolic. Thus, abbreviating the space $(M,-B)$ by $-M$, we have $\{-M\}=-\{M\}$ in $\mathrm{W}(\mathcal{O})$.

The bilinear form $B$ on $M$ extends in a unique way to a $K$-bilinear form $B^{\prime}$ on the $K$-vector space $E:=K \otimes_{\mathcal{O}} M$ obeying the formula

$$
B^{\prime}(c \otimes x, d \otimes y)=c d B(x, y)
$$

for $x, y \in M$ and $c, d \in K$. Identifying an element $x$ of $M$ with $1 \otimes x \in E$, we regard the free module $M$ as an $\mathcal{O}$-submodule of $E$. We then have $\left.B^{\prime}\right|_{M \times M}=B$. A basis $e_{1}, \ldots, e_{n}$ of $M$ over $\mathcal{O}$ is also a basis of $E$ over $K$, and the spaces $M$ and $E$ have the same symmetric matrix $\left(a_{i j}\right)$ with respect to $e_{1}, \ldots, e_{n}$. We often write 
$B$ instead of $B^{\prime}$.

If $U$ is a subspace of $E$, then $U \cap M$ is a subspace of $M$, and $K \cdot(U \cap M)=U$. In this way the subspaces of $E$ correspond uniquely to the subspaces of $M$. Clearly $U$ is totally isotropic iff $U \cap M$ is totally isotropic. Thus the following proposition is pretty obvious.

Proposition 3.1. Let $M$ be a space over $\mathcal{O}$ and $E:=K \otimes_{\mathcal{O}} M$.

a) $E$ is isotropic iff $M$ is isotropic.

b) $E$ is metabolic iff $M$ is metabolic.

It follows that the natural map $\{M\} \mapsto\left\{K \otimes_{\mathcal{O}} M\right\}$ from $W(\mathcal{O})$ to $\mathrm{W}(K)$, which is a ring homomorphism, is injective. We will often regard $\mathrm{W}(\mathcal{O})$ as a subring of $\mathrm{W}(K)$.

The square class group $Q(\mathcal{O}):=\mathcal{O}^{*} / \mathcal{O}^{* 2}$ of $\mathcal{O}$ injects into $Q(K)=K^{*} / K^{* 2}$ since clearly every unit of $\mathcal{O}$ which is a square in $K$ is a square in $\mathcal{O}$. As previously in the case of fields we identify a square class $a \mathcal{O}^{* 2}, a \in \mathcal{O}^{*}$, with the one-dimensional space $\langle a\rangle$ over $\mathcal{O}$ (more precisely, with its isometry class), and then observe that the natural map $Q(\mathcal{O}) \rightarrow \mathrm{W}(\mathcal{O})$ is injective, due to a natural commuting square

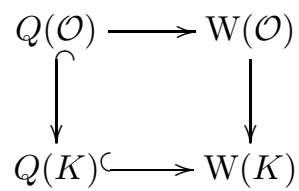

Thus $Q(\mathcal{O})$ can - and will - be also viewed as a subgroup of $\mathrm{W}(\mathcal{O})^{*}$. In other terms, if $a, b \in \mathcal{O}^{*}$ then $\langle a\rangle \sim\langle b\rangle$ iff $\langle a\rangle \cong\langle b\rangle$.

Without invoking the commutative square (7) this can be also proved by use of the signed determinant

$$
d(M):=\left\langle(-1)^{\frac{n(n-1)}{2}} \operatorname{det}\left(a_{i j}\right)\right\rangle
$$

of an n-dimensional space $M \cong\left(a_{i j}\right)$ over $\mathcal{O}$.

We switch to a place $\lambda: K \rightarrow L \cup \infty$ with valuation domain $\mathcal{O}=\mathcal{O}_{\lambda}$. The notation from $\S 2\left(\lambda_{W}\right.$, good reduction, $\lambda_{*}$ etc. $)$ will be freely used for spaces instead of forms.

Our place $\lambda$ restricts to a ring homomorphism $\left.\lambda\right|_{\mathcal{O}}$ from $\mathcal{O}$ to $L$, and $\left.\lambda\right|_{\mathcal{O}}$ factors through a field embedding $\bar{\lambda}: k \hookrightarrow L$. The definition of good reduction (Def. 2.1) and specialization under $\lambda$ now reads as follows.

Scholium 3.2. A bilinear space $E$ over $K$ has good reduction under $\lambda$ iff $E \cong$ $K \otimes_{\mathcal{O}} M$ for some bilinear space $M$ over $\mathcal{O}$. In this case

$$
\lambda_{*}(E) \approx L \otimes_{\lambda} M=L \otimes_{\bar{\lambda}} \bar{M} .
$$


Here $L \otimes_{\lambda} M$ denotes the scalar extension of the bilinear module $M$ to $L$ via $\lambda \mid \mathcal{O},{ }^{2}$ and $\bar{M}$ is the bilinear space $M / \mathfrak{m} M$ over $k$ obtained from $M$ by reduction modulo $\mathfrak{m}$.

Example 3.3. Every metabolic space over $K$ has good reduction. This follows easily from the fact that, for any $a, c \in K$ we have $\left(\begin{array}{cc}a & 1 \\ 1 & 0\end{array}\right) \cong\left(\begin{array}{cc}a c^{2} & 1 \\ 1 & 0\end{array}\right)$.

Corollary 2.7 tells us that, if $E$ and $F$ are spaces with good reduction and $E \approx F$, then $\lambda_{*}(E) \approx \lambda_{*}(F)$. $\left\{\right.$ In particular $\lambda_{*}(E)$ is well defined up to stable isometry. $\}$. This can now be proved in another, more transparent way as follows.

Let $E \cong K \otimes_{\mathcal{O}} M, F \cong K \otimes_{\mathcal{O}} N$ with spaces $M, N$ over $\mathcal{O}$. Then $K \otimes_{\mathcal{O}}(M \perp$ $-N) \cong E \perp-F$ is metabolic, hence $M \perp-N$ is metabolic by Proposition 3.1, and this implies that

$$
L \otimes_{\lambda}(M \perp-N) \cong L \otimes_{\lambda} M \perp\left(-L \otimes_{\lambda} N\right)
$$

is metabolic. Thus $L \otimes_{\lambda} M$ ad $L \otimes_{\lambda} N$ are Witt equivalent. Since these spaces have the same dimension, they are stably isomorphic.

We also want to describe the map $\lambda_{W}$ from $\S 2$ in geometric language.

Preparing for this we add more notation, which will be important also for later sections.

We choose a surjective valuation $v: K \rightarrow \Gamma \cup \infty$, essentially unique, associated with our valuation domain $\mathcal{O}$. So $\Gamma \cong K^{*} / \mathcal{O}^{*}$. \{ We use additive notation for $\Gamma$, so $v(x y)=v(x)+v(y)$.$\} We regard Q(\mathcal{O})$ as a subgroup of $Q(K)$, and we choose a complement $\Sigma$ of $Q(\mathcal{O})$ in $Q(K)$, i.e., a subgroup $\Sigma$ of $Q(K)$ with $Q(K)=Q(\mathcal{O}) \times \Sigma$.

This is possible, since the group $Q(K)$ is elementary abelian of exponent 2. Further, we choose, for every square class $\sigma \in \Sigma$ an element $s \in \mathcal{O}$ with $\sigma=\langle s\rangle$. For $\sigma=1$ we choose the representative $s=1$. Let $S$ be the set of these elements $s$. For every $a \in K^{*}$, there exists exactly one $s \in S$ and elements $\varepsilon \in \mathcal{O}^{*}, b \in K^{*}$ with $a=s \varepsilon b^{2}$. Since $K^{*} / \mathcal{O}^{*} \cong \Gamma$, it is clear that $S$ (resp. $\Sigma$ ) is a system of representatives of $\Gamma / 2 \Gamma$ in $K^{*}(\operatorname{resp} . Q(K))$ for the homomorphism from $K^{*}(\operatorname{resp} . Q(K))$ onto $\Gamma / 2 \Gamma$ determined by $v: K^{*} \rightarrow \Gamma$.

Definition 3.4. A $\lambda$-modular decomposition of a bilinear space $E$ over $K$ is an orthogonal decomposition

$$
E \cong \underset{s \in S}{\perp}\langle s\rangle \otimes\left(K \otimes_{\mathcal{O}} M_{s}\right)
$$

with every $M_{s}$ a space over $\mathcal{O}$ and only finitely many $M_{s} \neq 0$. Here the unadorned $\otimes$ means tensor product over $K$. Instead of " $\lambda$-modular" we also use the word "O-modular", since not the place $\lambda$ but only the valuation domain $\mathcal{O}$ is involved.

Every space $E$ over $K$ has a $\lambda$-modular decomposition. Indeed, we may decompose $E$ orthogonally in one-dimensional spaces and metabolic planes, usually in

\footnotetext{
${ }^{2}$ The bilinear form of $L \otimes_{\lambda} M$ is defined by a formula analogous to (6) above.
} 
many ways. One-dimensional spaces are products $\langle s\rangle \otimes\langle\varepsilon\rangle$ with $s \in S, \varepsilon \in \mathcal{O}^{*}$, and metabolic spaces are orthogonal sums of forms $\left(\begin{array}{cc}a & 1 \\ 1 & 0\end{array}\right)$ with $a \in \mathcal{O}$. One then simply gathers summands belonging to the same $s \in S$.

The following is now obvious from $\S 2$.

Scholium 3.5. Assume that

$$
E \cong \underset{s \in S}{\perp}\langle s\rangle \otimes\left(K \otimes_{\mathcal{O}} M_{s}\right)
$$

is a $\lambda$-modular decomposition of a space $E$ over $K$. Then

$$
\lambda_{W}(\{E\})=\left\{L \otimes_{\lambda} M_{1}\right\}
$$

In particular the space $L \otimes_{\mathcal{O}} M_{1}$ over $L$ is uniquely determined by $E$ up to Witt equivalence. In contrast to Scholium 3.2 we do not have a proof of this fact in simple geometric terms. Thus we cannot assert that the present "geometric language" supersedes the "algebraic language" of $\S 2$. We call the space $L \otimes_{\mathcal{O}} M_{1}$ a weak specialization of $E$ with respect to $\lambda$.

We add an important result about good reduction. Starting from now we often abbreviate "good reduction" by "GR".

First notice the trivial fact, that, if $E$ and $F$ are spaces over $K$ with GR under $\lambda$, then $E \perp F$ has again GR under $\lambda$, and

$$
\lambda_{*}(E \perp F) \approx \lambda_{*}(E) \perp \lambda_{*}(F) .
$$

Theorem 3.6. Let $E$ and $F$ bilinear spaces over $K$. Assume that $F$ and $E \perp F$ have GR under $\lambda$. Then $E$ has GR under $\lambda$.

Proof. Adding $-F$ to the space $F$ we retreat to the case that $F$ is metabolic. Let $E \perp F \cong K \otimes_{\mathcal{O}} N$ with $N$ a space over $\mathcal{O}$. We choose decomposition $E \cong E_{0} \perp E_{1}$ and $N \cong N_{0} \perp N_{1}$ with $E_{0}$ and $N_{0}$ anisotropic, $E_{1}$ and $N_{1}$ metabolic.

Then

$$
E_{0} \perp E_{1} \perp F \cong K \otimes_{\mathcal{O}} N_{0} \perp K \otimes_{\mathcal{O}} N_{1} .
$$

The spaces $E_{0}$ and $K \otimes_{\mathcal{O}} N_{0}$ are anisotropic, and the spaces $E_{1} \perp F$ and $K \otimes_{\mathcal{O}} N_{1}$ are metabolic. We conclude by Proposition 2.3 that $E_{0} \cong K \otimes_{\mathcal{O}} N_{0}$. Thus $E_{0}$ has GR. The space $E_{1}$ is metabolic, hence also has GR. Thus $E \cong E_{0} \perp E_{1}$ has GR.

\section{Quadratic modules}

We retain the notation and conventions of $\S 3$. In particular, $\mathcal{O}$ denotes a valuation domain, and modules over $\mathcal{O}$ will be free of finite rank, if nothing else is said.

A quadratic module $M=(M, q)$ over $\mathcal{O}$ is an $\mathcal{O}$-module $M$ equipped with a quadratic form $q$. This is a function $q: M \rightarrow \mathcal{O}$ such that $q(c x)=c^{2} q(x)$ for $c \in \mathcal{O}, x \in M$, and the map $B_{q}: M \times M \rightarrow \mathcal{O}$ given by

$$
B_{q}(x, y)=q(x+y)-q(x)-q(y)
$$


is $\mathcal{O}$-bilinear. If $e_{1}, \ldots, e_{n}$ is a basis of $M$ then $q$ is determined by the values $a_{i}=q\left(e_{i}\right), a_{i j}=B\left(e_{i}, e_{j}\right)$ for $i \neq j$. More precisely,

$$
q\left(\sum_{1}^{n} x_{i} e_{i}\right)=\sum_{i=1}^{n} a_{i} x_{1}^{2}+\sum_{i<j} a_{i j} x_{i} x_{j}
$$

for $x_{1}, \ldots, x_{n} \in \mathcal{O}$.

We have an obvious notion of isometry (= isomorphism) between quadratic module over $\mathcal{O}$, and most often will be only interested in the isometry class of a quadratic module $(M, q)$. Slightly abusively we abbreviate a quadratic module $M$ with quadratic form (13) by the symmetric matrix $\left[a_{i j}\right]$ in square brackets, with $a_{i j}$ as above ad $a_{i i}:=a_{i}$. The associated bilinear module $\widetilde{M}:=\left(M, B_{q}\right)$ is described by the matrix $\left(b_{i j}\right)$ with $b_{i i}=2 a_{i}$, and $b_{i j}=a_{i j}$ for $i \neq j$.

The orthogonal sum of two quadratic modules $\left(M_{1}, q_{1}\right)$ and $\left(M_{2}, q_{2}\right)$ over $\mathcal{O}$ is defined by

$$
\left(M_{1}, q_{1}\right) \perp\left(M_{2}, q_{2}\right):=\left(M_{1} \oplus M_{2}, q_{1} \perp q_{2}\right)
$$

with

$$
\left(q_{1} \perp q_{2}\right)\left(x_{1}+x_{2}\right):=q_{1}\left(x_{1}\right)+q_{2}\left(x_{2}\right)
$$

$\left(x_{1} \in M_{1}, x_{2} \in M_{2}\right)$. Notice that the associated bilinear module of $\left(M_{1}, q_{1}\right) \perp$ $\left(M_{2}, q_{2}\right)$ is the orthogonal sum $\widetilde{M}_{1} \perp \widetilde{M}_{2}$.

Orthogonality in a quadratic module $M=(M, q)$ refers to the bilinear form $B_{q}$. In particular, if $N_{1}$ and $N_{2}$ are submodules of $M$, then $M=N_{1} \perp N_{2}$ means that $M=N_{1} \oplus N_{2}$ as an $\mathcal{O}$-module and $B_{q}\left(N_{1}, N_{2}\right)=0$. The following fact will be used frequently.

Lemma 4.1. Let $M=(M, q)$ be a quadratic module. Assume that $N$ is a submodule of $M$ and the bilinear form $\left.B_{q}\right|_{N \times N}$ is non-degenerate. Then

$$
M=N \oplus N^{\perp} \text {. }
$$

Often we will denote a quadratic module by one letter, say $M$, without specifying the quadratic form on $M$. We then usually denote this form by $q$ and the associated bilinear form $B_{q}$ by $B$.

If $2 \neq 0$ in $\mathcal{O}$, then a quadratic module $M$ may be viewed as a bilinear module with $B(x, x) \in 2 \mathcal{O}$ for every $x \in M$ via the formula $B(x, x)=2 q(x)$, and if 2 is a unit of $\mathcal{O}$ we may identify in this way quadratic and bilinear modules over $\mathcal{O}$. But, if $2=0$ in $\mathcal{O}$, bilinear and quadratic modules over $\mathcal{O}$ are rather different objects.

\section{Definition 4.2.}

a) A quadratic module $N=(N, q)$ is called quasilinear if $B_{q}=0$.

b) If $M$ is any quadratic module over $\mathcal{O}$, then the quadratic module

$$
M^{\perp}:=\{x \in M \mid B(x, M)=0\}
$$

with the form $\left.q\right|_{M^{\perp}}$ is called the quasilinear part of $M$. We denote it by $Q L(M)$. 
$M^{\perp}$ is a direct summand of the $\mathcal{O}$-module $M$. Choosing any submodule $N$ of $M$ with $M=N \oplus M^{\perp}$ we have

$$
M \cong N \perp Q L(M)
$$

as quadratic module. Moreover, $Q L(M)$ is an orthogonal sum of quadratic modules of dimension $(=\operatorname{rank}) 1$,

$$
Q L(M)=\left[a_{1}\right] \perp \ldots \perp\left[a_{n}\right]
$$

with $a_{i} \in \mathcal{O}$.

The following definition will be central for our theory of good reduction and specialization of a quadratic form over a field under a place.

Definition 4.3. We call a quadratic module $(M, q)$ over $\mathcal{O}$ nondegenerate, if it satisfies the following conditions:

(Q0) $M$ is free of finite rank.

(Q1) The bilinear form $\overline{B_{q}}$, induced by $B_{q}$ on $M / M^{\perp}$ in the obvious way, is nondegenerate.

(Q2) $q(x) \in \mathcal{O}^{*}$ for every vector $x$ in $M^{\perp}$, which is primitive in $M^{\perp}$ (and hence in $M)$.

If instead of (Q2), the following condition is satisfied

$\left(\mathrm{Q} 2^{\prime}\right) Q L(M)=0$ or $Q L(M) \cong[\varepsilon]$ with $\varepsilon \in \mathcal{O}^{*}$,

then we call $(M, q)$ regular.

In the special case $M^{\perp}=0$, we call $(M, q)$ strictly regular.

Comment on conditions (Q2) and (Q2').

If $2 \neq 0$ in $\mathcal{O}$, then $\left.q\right|_{M^{\perp}}=0$ and the requirement (Q2) implies $M^{\perp}=0$, hence implies - in conjunction with (Q0) and (Q1) - strict regularity. If $2 \in \mathcal{O}^{*}$, then the nondegenerate quadratic $\mathcal{O}$-modules are the same objects as the nondegenerate bilinear $\mathcal{O}$-modules, as defined in $\S 3$.

Suppose now that $2=0$ in $\mathcal{O}$. The condition $M^{\perp}=0$, in other words, strict regularity, is very natural but too limited for applications. Indeed, if $M^{\perp}=0$, then the bilinear module $\left(M, B_{q}\right)$ is nondegenerate and we have $B_{q}(x, x)=2 q(x)=0$ for every $x \in M$. This implies that $M$ has even dimension, as is well-known. (To prove this, consider the bilinear space $K \otimes_{\mathcal{O}} \widetilde{M}$.) So, if we insist on using strict regularity, we can only deal with quadratic forms of even dimension.

On the other hand, property Q2 has an annoying effect: Q2 is not always preserved under a base extension. If $\mathcal{O}^{\prime} \supset \mathcal{O}$ is another valuation domain, whose maximal ideal $\mathfrak{m}^{\prime}$ lies over $\mathfrak{m}$, i.e., $\mathfrak{m}^{\prime} \cap \mathcal{O}=\mathfrak{m}$, and if $M$ is non degenerate, then $\mathcal{O}^{\prime} \otimes_{\mathcal{O}} M$ can be degenerate. However, if $M$ satisfies $\left(\mathrm{Q} 2^{\prime}\right)$, this clearly cannot happen.

In the case $\mathfrak{m}=0$, i.e., $\mathcal{O}=K$, we call a non degenerate quadratic $\mathcal{O}$-module a quadratic space over $K$.

We gather some facts about nondegenerate quadratic modules, all to be found in [Spez, §6]. In the following $M=(M, q)$ is a quadratic module over $\mathcal{O}$. 
Fact 4.4. If $M$ is nondegenerate then $M$ is an orthogonal sum of quadratic modules $\left[\begin{array}{ll}\alpha & 1 \\ 1 & \beta\end{array}\right]$, with $\alpha, \beta \in \mathcal{O}, 1-4 \alpha \beta \in \mathcal{O}^{*}$, and modules $[\varepsilon]$ with $\varepsilon \in \mathcal{O}^{*}$.

Fact 4.5. If $M$ is regular and $\operatorname{dim} M$ is even, then $M$ is strictly regular and equal to an orthogonal sum of modules $\left[\begin{array}{cc}\alpha & 1 \\ 1 & \beta\end{array}\right]$, with $\alpha, \beta \in \mathcal{O}, 1-4 \alpha \beta \in \mathcal{O}^{*}$.

Fact 4.6. Assume that $M$ is nondegenerate. Then every primitive vector $e \in M$ with $q(e)=0$ can be completed to a hyperbolic vector pair, i.e., a pair e,f with $q(f)=0$ and $B(e, f)=1$.

As an illustration, how our conditions Q1 and Q2 can be put to work, we give the proof of 4.6. We choose a decomposition $M=N \perp M^{\perp}$ and write $e=x+y$ with $x \in N, y \in M^{\perp}$. Suppose for the sake of contradiction that the vector $x$ is not primitive in $N$, hence not primitive in $M$. Then $y$ is primitive in $M^{\perp}$, and thus $q(y) \in \mathcal{O}^{*}$ by condition Q2. Hence also $q(x)=-q(y) \in \mathcal{O}^{*}$ and $x$ has to be primitive, a contradiction.

Thus $x$ is primitive in $N$. Since $B_{q}$ is nondegenerate on $N$, there exists some $z \in N$ with $B_{q}(x, z)=1$. We also have $B_{q}(e, z)=1$. Clearly $f:=z-q(z) e$ completes the vector $e$ to a hyperbolic pair.

Fact 4.7. (Cancellation theorem) If $M$ and $N$ are quadratic $\mathcal{O}$-modules and $G$ is a strictly regular quadratic $\mathcal{O}$-module with $M \perp G \cong N \perp G$, then $M \cong N$.

We call a quadratic $\mathcal{O}$-module $M$ isotropic, if $M$ contains a vector $x \neq 0$ with $q(x)=0$, anisotropic otherwise. We call $M$ hyperbolic if $M$ is isometric to an orthogonal sum $r \times\left[\begin{array}{ll}0 & 1 \\ 1 & 0\end{array}\right]$ of $r$ copies of the "hyperbolic plane" $\left[\begin{array}{ll}0 & 1 \\ 1 & 0\end{array}\right]$ for some $r \in \mathbb{N}$. As in the bilinear case one proves easily:

Fact 4.8. $M$ is isotropic iff the quadratic module $K \otimes_{\mathcal{O}} M$ over $K$ is isotropic, and $M$ is hyperbolic iff $K \otimes_{\mathcal{O}} M$ is hyperbolic.

Fact 4.9. (Witt decomposition). If $M$ is nondegenerate, then

$$
M \cong M_{0} \perp r \times\left[\begin{array}{ll}
0 & 1 \\
1 & 0
\end{array}\right]
$$

with $M_{0}$ anisotropic (and nondegenerate) and $r \in \mathbb{N}_{0}$.

It follows from 4.7 that the number $r$ and the isometry class of $M_{0}$ are uniquely determined by $M$. We call $r$ the (Witt-) index of $M$ and $M_{0}$ the kernel module, or the anisotropic part of $M$, and we write $r=\operatorname{ind}(M), M_{0}=\operatorname{ker}(M)$. We often denote the hyperbolic plane $\left[\begin{array}{ll}0 & 1 \\ 1 & 0\end{array}\right]$ by $H$ (regardless which ring $\mathcal{O}$ is under consideration).

Notice also that, by 4.8 , we have $\operatorname{ind}\left(K \otimes_{\mathcal{O}} M\right)=\operatorname{ind}(M)$ and $\operatorname{ker}\left(K \otimes_{\mathcal{O}} M\right)=$ $K \otimes_{\mathcal{O}} \operatorname{ker}(M)$.

Fact 4.10. If $M=(M, q)$ is strictly regular then

$$
(M, q) \perp(M,-q) \cong(\operatorname{dim} M) \times H .
$$


If we write $M$ for $(M, q)$ we usually write $-M$ for $(M,-q)$, following the same practice as for bilinear modules.

Definition 4.11. We call two nondegenerate quadratic modules $M$ and $N$ over $\mathcal{O}$ Witt-equivalent, and write $M \sim N$ if there exist natural numbers $s, t$ such that

$$
M \perp s \times\left[\begin{array}{ll}
0 & 1 \\
1 & 0
\end{array}\right] \cong N \sim t \times\left[\begin{array}{ll}
0 & 1 \\
1 & 0
\end{array}\right] .
$$

Due to 4.9 this happens iff $\operatorname{ker}(M) \cong \operatorname{ker}(N)$. The Witt class of $M$, i.e., the equivalence of $M$ under $\sim$, will be denoted by $\{M\}$.

It is now easy to verify:

Fact 4.12. If $M, M^{\prime}$ are strictly regular and $N, N^{\prime}$ are non degenerate quadratic modules over $\mathcal{O}$ with $M \sim M^{\prime}$ and $N \sim N^{\prime}$, then $M \perp N$ and $M^{\prime} \perp N^{\prime}$ are non degenerate and $M \perp N \sim M^{\prime} \perp N^{\prime}$.

Definition 4.13. We denote the set of Witt classes of nondegenerate quadratic $\mathcal{O}$ modules by $\widetilde{\mathrm{Wq}}(\mathcal{O})$. We denote the subsets of Witt classes of regular, resp. strictly regular quadratic $\mathcal{O}$-modules by $\operatorname{Wqr}(\mathcal{O})$, resp. $\operatorname{Wq}(\mathcal{O})$.

Due to 4.12 we have a well-defined "addition" of classes $\{M\} \in \mathrm{Wq}(\mathcal{O})$ with classes $\{N\} \in \widetilde{\mathrm{Wq}}(\mathcal{O})$,

$$
\{M\}+\{N\}:=\{M \perp N\} .
$$

Restricting also $\{N\}$ to $\mathrm{Wq}(\mathcal{O})$ we obtain on $\mathrm{Wq}(\mathcal{O})$ the structure of an abelian group. This groups $\mathrm{Wq}(\mathcal{O})$ operates by addition on the set $\widetilde{\mathrm{Wq}}(\mathcal{O})$. The subset $\operatorname{Wqr}(\mathcal{O})$ is a union of orbits.

Definition 4.14. We call $\mathrm{Wq}(\mathcal{O})$ the quadratic Witt group of $\mathcal{O}, \widetilde{\mathrm{Wq}}(\mathcal{O})$ the quadratic Witt set of $\mathcal{O}$, and $\operatorname{Wqr}(\mathcal{O})$ the regular quadratic Witt set of $\mathcal{O}$.

In the case $\mathcal{O}=K$ we go further and define Witt classes of arbitrary (finite dimensional) quadratic modules over $K$ as follows.

Starting with such a module $M=(M, q)$ we define the "defect" of $M$ by

$$
\delta(M)=\left\{x \in M^{\perp}: q(x)=0\right\} .
$$

The form $q$ gives us a quadratic form $\bar{q}$ on $M / \delta(M)$ in the obvious way, hence a quadratic space $(M / \delta(M), \bar{q})$, which we call the quadratic space associated to $M$ and denote by $\widehat{M}$. Clearly

$$
M \cong \widehat{M} \perp \delta(M) \cong \widehat{M} \perp s \times[0]
$$

with $s:=\operatorname{dim} \delta(M)$. We have

$$
M \cong M_{0} \perp r \times H
$$

with $\widehat{M}_{0}$ anisotropic and $r \in \mathbb{N}_{0}$.

Again $M_{0}$ and $r$ are uniquely determined by $M$, and again we call $M_{0}$ the kernel module $\operatorname{ker}(M)$ of $M$ and $r$ the index $\operatorname{ind}(M)$ of $M$. Notice that now $M_{0}$ can be isotropic. Notice that in contrast to strictly regular quadratic spaces we do not have cancellation in general. For example $[a] \perp[a] \cong[0] \perp[a]$ for any $a \in K$. 
We call two quadratic modules $M, N$ over $K$ Witt equivalent, and write $M \sim N$, if $M \perp s \times H \cong N \perp t \times H$ for some number $s, t$. This means the same as $\operatorname{ker}(M) \cong \operatorname{ker}(N)$. We denote the set of Witt equivalence classes $\{M\}$ of quadratic $K$-modules $M$ by $\widehat{\mathrm{Wq}}(K)$, and we call $\widehat{\mathrm{Wq}}(K)$ the defective quadratic Witt set of $K$. The value $\{M\}+\{N\}=\{M \perp N\}$ makes $\widehat{\mathrm{Wq}}(K)$ an abelian semigroup with neutral element $\{0\}=\{H\}$. It contains $\widetilde{\mathrm{Wq}}(K)$ as a subset and $\mathrm{Wq}(K)$ as a subgroup.

The reason why we need $\widehat{\mathrm{Wq}}(K)$ instead of just $\widetilde{\mathrm{Wq}}(K)$ is lack of functoriality of the latter set. If $K^{\prime} \supset K$ is a field extension, we have a well defined semigroup homomorphism $\widehat{\mathrm{Wq}}(K) \rightarrow \widehat{\mathrm{Wq}}\left(K^{\prime}\right)$ mapping a class $\{M\}$ to $\left\{K^{\prime} \otimes_{K} M\right\}$. This homomorphism does not map $\widetilde{\mathrm{Wq}}(K)$ to $\widetilde{\mathrm{Wq}}\left(K^{\prime}\right)$ in general (if char $K=2$ ), since for a space $M$ over $K$ the quasilinear part of $K^{\prime} \otimes_{K} M$ may be isotropic.

We return to an arbitrary valuation $\operatorname{ring} \mathcal{O}$. If $M_{1}=\left(M_{1}, B_{1}\right)$ is a bilinear $\mathcal{O}$ module and $M_{2}=\left(M_{2}, q_{2}\right)$ is a quadratic $\mathcal{O}$-module, we can install on the tensor product $M_{1} \otimes_{\mathcal{O}} M_{2}$ a quadratic form $q:=B_{1} \otimes q_{2}$ be choosing a (non symmetric) bilinear form $\beta_{2}$ with $\beta_{2}(z, z)=q_{2}(z)$ for all $z \in M_{2}$, taking the tensor product $\beta:=B_{1} \otimes \beta_{2}$ on $M_{1} \otimes M_{2}$, and putting $q(x):=\beta(x, x)$. The quadratic form $q$ is independent of the choice of $\beta$, and can be characterized by the rules

$$
B_{q}=B_{1} \otimes B_{q_{2}}, \quad q\left(x_{1} \otimes x_{2}\right)=B_{1}\left(x_{1}, x_{1}\right) q_{2}\left(x_{2}\right)
$$

$\left(x_{1} \in M_{1}, x_{2} \in M_{2}\right)$.

We denote the quadratic module $\left(M_{1} \otimes_{\mathcal{O}} M_{2}, q\right)$ by $M_{1} \otimes M_{2}$ for short. If $M_{1}=\left\langle a_{1}, \ldots, a_{n}\right\rangle$, then

$$
M_{1} \otimes M_{2} \cong\left(M_{2}, a_{1} q_{2}\right) \perp \ldots \perp\left(M_{2}, a_{r} q_{2}\right) .
$$

In particular, for any $a \in K$,

$$
\langle a\rangle \otimes M_{2} \cong\left(M_{2}, a q_{2}\right) .
$$

If $M_{1}$ is non degenerate and $M_{2}$ is strictly regular then $M_{1} \otimes M_{2}$ is strictly regular. It is now straightforward to verify that we have a well defined product of Witt classes

$$
\left\{M_{1}\right\} \cdot\left\{M_{2}\right\}=\left\{M_{1} \otimes M_{2}\right\},
$$

which turns $\mathrm{Wq}(\mathcal{O})$ into a module over the ring $\mathrm{W}(\mathcal{O})$. \{ Notice in particular that, if $M_{1} \approx M_{1}^{\prime}$ then $M_{1} \otimes M_{2} \cong M_{1}^{\prime} \otimes M_{2}$. $\}$ Unfortunately there seems to be no good way to let $\mathrm{W}(\mathcal{O})$ operate on $\widetilde{\mathrm{Wq}}(\mathcal{O})$.

\section{WEAK SPECIALIZATION AND GOOD REDUCTION}

As in previous sections $\lambda: K \rightarrow L \cup \infty$ is a place, $\mathcal{O}=\mathcal{O}_{\lambda}$ is the valuation domain of $\lambda, \mathfrak{m}$ its maximal ideal and $k=\mathcal{O} / \mathfrak{m}$ its residue class field. Let $E=(E, q)$ be a quadratic space over $K$.

Definition 5.1. We say that $E$ has good reduction (abbreviated: GR) with respect to $\lambda$ if $E \cong K \otimes_{\mathcal{O}} M$ with $M$ a non degenerate quadratic $\mathcal{O}$-module. 
In this situation we obtain from $E$ a quadratic $L$-module

$$
\lambda_{*}(E):=L \otimes_{\lambda} M=L \otimes_{\bar{\lambda}} M / \mathfrak{m} M
$$

(Notations analogous to those in $\S 3$ ). Notice that the "reduced" quadratic module $M / \mathfrak{m} M$ over $k$ is non degenerate, but $L \otimes_{\bar{\lambda}} M / \mathfrak{m} M$ may be degenerate, since the quasilinear part of $M / \mathfrak{m} M$ may become isotropic over $L$.

We would like to prove that the quadratic module $\lambda_{*}(E)$ is independent of the choice of $M$.

Only then the notation $\lambda_{*}(E)$ will be justified. If $E$ is strictly regular this can be done by the same sort of geometric argument as used in $\S 3$ in the bilinear case. To prove it in the general case we would like to use an additive map $\lambda_{W}: \widehat{\mathrm{Wq}}(K) \rightarrow \widehat{\mathrm{Wq}}(L)$, similar to the map $\lambda_{W}: \mathrm{W}(K) \rightarrow \mathrm{W}(L)$ from $\S 2$, and then to proceed in a similar way as in $\S 2$ and $\S 3$ for bilinear spaces. But now a new path has to be taken, since we do not have a presentation of $\widehat{\mathrm{Wq}}(K)$ by generators and relations which fits well with the place $\lambda$.

Let $\mathcal{O}^{h}$ denote the henselization of $\mathcal{O}, K^{h}$ its field of quotient (= the henselization of $K$ with respect to $\mathcal{O}$ ). $\lambda$ extends to a place $\lambda^{h}: K^{h} \rightarrow L \cup \infty$ with valuation ring $\mathcal{O}^{h}$, since $\mathcal{O}^{h}$ has the same residue class field $\mathcal{O}^{h} / \mathfrak{m}^{h}=\mathcal{O} / \mathfrak{m}$ as $\mathcal{O}$. If $M$ is a non degenerate quadratic $\mathcal{O}$-module, $M^{h}:=\mathcal{O}^{h} \otimes_{\mathcal{O}} M$ is again non degenerate and

$$
L \otimes_{\lambda} M=L \otimes_{\lambda^{h}} M^{h} .
$$

Thus we can retreat to the case that $\mathcal{O}$ is henselian.

Here the following lemma offers help.

Lemma 5.2. Assume that $\mathcal{O}$ is henselian. Let $E=(E, q)$ be an anisotropic quadratic space over $K$.

(a) The sets

$$
\mu(E):=\{x \in E \mid q(x) \in \mathcal{O}\} \text { and } \mu_{+}(E):=\{x \in E \mid q(x) \in \mathfrak{m}\}
$$

are $\mathcal{O}$-submodules of $E$.

(b) For any $x \in \mu(E)$ and $y \in \mu_{+}(E)$ we have $q(x+y)-q(x) \in \mathfrak{m}$ and $B_{q}(x, y) \in \mathfrak{m}$

By this lemma

$$
\rho(E):=\mu(E) / \mu_{+}(E)
$$

is a $k$-vector space in a natural sense $(k=\mathcal{O} / \mathfrak{m})$. We define a function $\bar{q}: \rho(E) \rightarrow k$ as follows:

$$
\bar{q}(\bar{x}):=\overline{q(x)} \quad(x \in \mu(E))
$$

where $\bar{x}$ denotes the image of $x \in \mu(E)$ in $\rho(E)$ and $\bar{a}$ denotes the image of $a \in \mathcal{O}$ in $k$. Lemma 4.1 tells us that the map $\bar{q}$ is well defined, and, using the lemma further, one proves easily that $\bar{q}$ is a quadratic form on the $k$-vector space $\rho(E)$ with associated bilinear form $\bar{B}=B_{\bar{q}}$ given by

$$
\bar{B}(\bar{x}, \bar{y})=\overline{B(x, y)} \text {. }
$$


The quadratic $k$-module $(\rho(E), \bar{q})$ is clearly anisotropic.

If $\mathcal{O}$ is not necessarily henselian we are motivated by this lemma to make the following Ansatz in order to associate to a space $E$ over $K$ a Witt class $\lambda_{W}\{E\}$ over $L$ :

$$
\lambda_{W}\{E\}:=\left\{L \otimes_{\bar{\lambda}} \rho\left(\operatorname{ker}\left(K^{h} \otimes E\right)\right\},\right.
$$

where, as before, $\bar{\lambda}: k \hookrightarrow L$ is the field embedding determined by $\lambda$.

All good and well, if only we know whether the vector space

$$
\rho\left(\operatorname{ker}\left(K^{h} \otimes E\right)\right)
$$

has finite dimension! To guarantee this we have to confine the class of allowed quadratic modules $E$.

As explicated in $\S 3$ we choose a system $S$ of representatives of $\Gamma / 2 \Gamma$ in $K$ (with $1 \in S$ ), where $\Gamma=K^{*} / \mathcal{O}^{*}$ is the value group of the natural valuation associated to $\mathcal{O}$.

Definition 5.3. A quadratic space $E$ over $K$ is obedient with respect to $\lambda$ if $E$ has an orthogonal decomposition

$$
E=\underset{s \in S}{\perp} E_{s}
$$

such that each space $\left(E_{s}, s \cdot\left(\left.q\right|_{E_{s}}\right)\right)$ has GR under $\lambda$, hence

$$
E_{s}=K M_{s} \cong\langle s\rangle \otimes\left(K \otimes \mathcal{O} M_{s}\right)
$$

with $M_{s}$ a non degenerate quadratic $\mathcal{O}$-submodule of $E_{s}{ }^{3}$ Then (16) is called a $\lambda$-modular decomposition of $E$, and (16), (17) is called a $\lambda$-modular representation of $E$. Instead of " $\lambda$-modular" we also use the term "OO-modular".

It is no big deal to verify the following two lemmas.

Lemma 5.4. Assume that $\mathcal{O}$ is henselian and $E$ is an obedient anisotropic quadratic space over $K$, with $\mathcal{O}$-modular representation $(16),(17)$. Then $\left(M_{1},\left.q\right|_{M_{1}}\right)$ is the only non degenerate quadratic $\mathcal{O}$-submodule of $E_{1}$, and

$$
\begin{aligned}
& \mu(E)=M_{1} \perp \underset{s \neq 1}{\perp} \mu_{+}\left(E_{s}\right), \\
& \mu_{+}(E)=\mathfrak{m} M_{1} \perp \underset{s \neq 1}{\perp} \mu_{+}\left(E_{s}\right) .
\end{aligned}
$$

Thus

$$
(\rho(E), \bar{q}) \cong\left(M_{1} / \mathfrak{m} M_{1}, \overline{q_{1}}\right)
$$

with $q_{1}:=\left.q\right|_{M_{1}}$.

\footnotetext{
${ }^{3}$ Of course, $E_{s} \neq 0$ only for finitely many $s \in S$.
} 
Lemma 5.5. Let $\mathcal{O}$ be henselian. Let $s_{1}, \ldots, s_{r}$ be different elements of $S$ and $M_{1}, \ldots, M_{r}$ anisotropic nondegenerate quadratic $\mathcal{O}$-modules. Then

$$
E:=\stackrel{n}{\perp}\left\langle s_{i}\right\rangle \otimes\left(K \otimes_{\mathcal{O}} M_{i}\right)
$$

is an anisotropic quadratic space over $K$.

We arrive at the main theorem of this section.

Theorem 5.6. Let $E$ be a quadratic space over $K$, obedient with respect to $\mathcal{O}$. Let

$$
E=\underset{s \in S}{\perp} E_{s}=\underset{s \in S}{\perp} F_{s}
$$

be two $\mathcal{O}$-modular decompositions of $E$, and also let $M_{1}, N_{1}$ be nondegenerate quadratic $\mathcal{O}$-modules with $E_{1} \cong K \otimes_{\mathcal{O}} M_{1}, F_{1} \cong K \otimes_{\mathcal{O}} N_{1}$. Then the quadratic spaces $M_{1} / \mathfrak{m} M_{1}$ and $N_{1} / \mathfrak{m} N_{1}$ over $k=\mathcal{O} / \mathfrak{m}$ are Witt equivalent.

For the proof one passes from $K$ to $K^{h}$, chooses Witt decompositions of the quadratic $\mathcal{O}^{h}$-modules $M_{i}^{h}, N_{j}^{h}$, and then computers the kernel space of $E^{h}$ in two different ways applying Lemma 5 . Then Lemma 4 gives the result.

Definition 5.7. Let $E$ be a quadratic space over $K$, obedient with respect to $\lambda$. If $E=\underset{s \in S}{\perp} E_{s}$ is a $\lambda$-modular decomposition of $E$, and $M_{1}$ is a non degenerate quadratic $\mathcal{O}$-module with $E_{1} \cong K \otimes_{\mathcal{O}} M_{1}$, then we call the quadratic space

$$
L \otimes_{\lambda} M_{1}=L \otimes_{\bar{\lambda}} M_{1} / \mathfrak{m} M_{1}
$$

a weak specialization of $E$ with respect to $\lambda$. (As before, $\otimes_{\lambda}$ denotes a base extension with respect to the homomorphism $\left.\lambda\right|_{\mathcal{O}}: \mathcal{O} \rightarrow L$.)

By Theorem 5.6, the space $L \otimes_{\lambda} M_{1}$ is uniquely determined by $E$ and $\lambda$, up to Witt equivalence. We denote its Witt class by $\lambda_{W}(E)$, i.e.,

("W" as in "Witt" or "weak".)

$$
\lambda_{W}(E):=\left\{L \otimes_{\lambda} M_{1}\right\} \in \widehat{\mathrm{Wq}}(L) .
$$

Remark. If $E$ is strictly regular, then $E_{1}$ is strictly regular, hence $M_{1}$ is strictly regular, and we conclude that $\lambda_{W}(E) \in \mathrm{Wq}(L)$. In particular this happens if $\operatorname{char} K \neq 2$. If $E$ is only regular then $M_{1}$ is still regular, hence $\lambda_{W}(E) \in \operatorname{Wqr}(L)$, since now the quasilinear part of $L \otimes_{\lambda} M_{1}$ has at most dimension 1, hence is anisotropic. If $\operatorname{char} L \neq 2$ then quadratic spaces over $K$ resp. $L$ can be identified with bilinear spaces over $K$ resp. $L$, and the present weak specialization coincides with the weak specialization of $\S 2$ and $\S 3$.

Corollary 5.8. If $E$ and $E^{\prime}$ are quadratic spaces over $K$, both obedient with respect to $\lambda$, and if $E \sim E^{\prime}$, then $\lambda_{W}(E)=\lambda_{W}\left(E^{\prime}\right)$.

Proof. This can be quickly deduced from Theorem 5.6. Suppose without loss of generality that $\operatorname{dim} E \leq \operatorname{dim} E^{\prime}$. Then $E^{\prime} \cong E \perp r \times\left[\begin{array}{ll}0 & 1 \\ 1 & 0\end{array}\right]$ for a certain $r \in \mathbb{N}_{0}$. If we choose a non degenerate $\mathcal{O}$-module $M_{1}$ for $E_{1}$, as in Definition 5.7, the 
$M_{1}^{\prime}:=M_{1} \perp r \times\left[\begin{array}{ll}0 & 1 \\ 1 & 0\end{array}\right]$ is a possible choice for $E^{\prime}$. Therefore, $L \otimes_{\lambda} M_{1}^{\prime} \sim L \otimes_{\lambda} M_{1}$.

Remark 5.9. Let $E$ and $F$ be quadratic spaces over $K$, obedient with respect to $\lambda$, and suppose that $E$ is strictly regular.

Obviously we then have

$$
\lambda_{W}(E \perp F)=\lambda_{W}(E)+\lambda_{W}(F) .
$$

\{The addition of an element of $\mathrm{Wq}(K)$ and an element of $\widetilde{\mathrm{Wq}}(K)$ has been explained in $\S 4$.

We do not exploit here the full power of weak specializations but use them only to justify the Ansatz (15) from the beginning of the section for specializing a space with good reduction.

Scholium 5.10. Assume that $E$ has $G R$ under $\lambda, E \cong K \otimes_{\mathcal{O}} M$ with $M$ a nondegenerate quadratic $\mathcal{O}$-module. Then $L \otimes_{\lambda} M$ is uniquely determined by $E$ and $\lambda$ up to isometry. Indeed, the Witt class of $L \otimes_{\lambda} M$ does not depend on the choice of $M$ by Theorem 5.6, and $\operatorname{dim} L \otimes_{\lambda} M=\operatorname{dim} E$.

Definition 5.11. If $E$ has GR under $\lambda$ we define

$$
\lambda_{*}(E):=L \otimes_{\lambda} M,
$$

and we call $\lambda_{*}(E)$ the specialization of $E$ under $\lambda$.

If $E$ and $F$ are quadratic $K$-spaces with GR under $\lambda$ and $E$ is strictly regular, then $E \perp F$ has GR under $\lambda$ and clearly

$$
\lambda_{*}(E \perp F)=\lambda_{*}(E) \perp \lambda_{*}(F) .
$$

By arguments analogous to the proof of Theorem 3.6 one now obtain the following important fact.

Theorem 5.12. Let $F$ and $G$ be quadratic spaces over $K$. Suppose that $F$ is strictly regular. If $F$ and $F \perp G$ have GR with respect to $\lambda$, then $G$ also has $G R$ with respect to $\lambda$.

We mention that - under some precaution - weak specialization is compatible with the tensor product of a bilinear and quadratic space. For example the following holds.

Remark 5.13. Let $F$ be a bilinear space and $G$ a strictly regular quadratic space our $K$. Suppose that $G$ has $G R$ under $\lambda$. Then $F \otimes G$ is obedient with respect to $\lambda$, and

$$
\lambda_{W}(F \otimes G)=\lambda_{W}(F) \lambda_{W}(G)=\lambda_{W}(F)\left\{\lambda_{*}(G)\right\} .
$$




\section{Generic SPLitTing OF QUADRATIC FORMS}

In the following quadratic $\mathcal{O}$-modules leave the stage and will act only from the background. Openly we only deal with quadratic spaces over fields. Thus we switch to the language of quadratic forms (= homogeneous polynomials of degree 2 ) over fields, freely using the terminology of $\S 4$ and $\S 5$ for forms instead of spaces.

If $\varphi=\varphi\left(x_{1}, \ldots, x_{n}\right)$ is a form ${ }^{4}$ over a field $k$ and $k \subset K$ is a field extension then $\varphi \otimes K$ denotes the polynomial $\varphi$ as an element of $K\left[x_{1}, \ldots, x_{n}\right]$ instead of $k\left[x_{1}, \ldots, x_{n}\right]$.

It turns out that we can extend the well known generic splitting theory of forms over fields of characteristic $\neq 2$ to arbitrary fields, as long as we can guarantee that under the relevant places $\lambda: K \rightarrow L \cup \infty$ a given form $\varphi$ over $K$ with GR with respect to $\lambda^{5}$ has a specialization $\lambda_{*}(\varphi)$ which is again nondegenerate, i.e., the specialization $\lambda_{*}(Q L(\varphi))$ of the quasilinear part $Q L(\varphi)$ remains anisotropic \{ Slogan: "Do not destroy the quasilinear part!"

Definition 6.1. Let $\varphi$ be a nondegenerate form over a field $k$. We call a field extensions $k \subset K \quad \varphi$-conservative if $K \otimes \varphi$ is again nondegenerate, i.e., $K \otimes Q L(\varphi)$ is anisotropic.

Notice that a separable field extension $k \subset K$ is $\varphi$-conservative for every $\varphi$, since an anisotropic quasilinear form over $k$ remains anisotropic over $K$.

Notice also that, if $\varphi$ is regular then every field extension $k \subset K$ is $\varphi$-conservative, since forms of dimension $\leq 1$ cannot become isotropic.

The generic splitting theory of a non degenerate form $\varphi$ over $k$ will deal with the Witt decomposition of $K \otimes \varphi$ for $K$ varying in the class of all $\varphi$-conservative field extension of $k$.

The following observation is crucial here.

Theorem 6.2. Let $\lambda: K \rightarrow L \cup \infty$ be a place and $\varphi$ a form over $K$ which has $G R$ with respect to $\lambda$. Suppose that also $\lambda_{*}(\varphi)$ is nondegenerate. Suppose further that $K^{\prime} \supset K$ is a field extension and that $\mu: K^{\prime} \rightarrow L \cup \infty$ is a place extending $\lambda$. Then the form $\varphi \otimes K^{\prime}$ has $G R$ with respect to $\mu$ and

$$
\mu_{*}\left(\varphi \otimes K^{\prime}\right)=\lambda_{*}(\varphi) .
$$

Proof. Let $\mathcal{O}:=\mathcal{O}_{\lambda}, \mathcal{O}^{\prime}:=\mathcal{O}_{\mu}$, and let $k$ and $k^{\prime}$ denote the residue class fields of $\mathcal{O}$ and $\mathcal{O}^{\prime}$ respectively. The field extension $\bar{\lambda}: k \hookrightarrow L$ is a composition of the extensions $k \hookrightarrow k^{\prime}$ and $\bar{\mu}: k^{\prime} \hookrightarrow L$, where the first extension is induced by the inclusion $\mathcal{O} \hookrightarrow \mathcal{O}^{\prime}$.

Let $E$ be a quadratic space for $\varphi$ and $M$ a nondegenerate quadratic $\mathcal{O}$-module with $E \cong K \otimes_{\mathcal{O}} M$. Then $K^{\prime} \otimes E=K^{\prime} \otimes_{\mathcal{O}^{\prime}} M^{\prime}$ with $M^{\prime}:=\mathcal{O}^{\prime} \otimes_{\mathcal{O}} M$. The

\footnotetext{
4 "Form" will always mean "quadratic form".

5 This assumption presupposes that $\varphi$ is nondegenerate (cf. $\S 5$, Def. 1).
} 
quasilinear quadratic $k$-module $G:=k \otimes_{\mathcal{O}} Q L(M)$ is anisotropic. By assumption, $L \otimes_{\bar{\lambda}} G=Q L\left(L \otimes_{\lambda} M\right)$ is also anisotropic. Therefore

$$
k^{\prime} \otimes_{k} G=k^{\prime} \otimes_{\mathcal{O}}, Q L\left(M^{\prime}\right)
$$

is anisotropic. This proves that $M^{\prime}$ is a nondegenerate quadratic $\mathcal{O}^{\prime}$-module. Hence $\varphi \otimes K^{\prime}$ is nondegenerate and has GR with respect to $\mu$. Furthermore $\mu_{*}\left(\varphi \otimes K^{\prime}\right)$ corresponds to the quadratic space

$$
L \otimes_{\mu} M^{\prime}=L \otimes_{\mu}\left(\mathcal{O}^{\prime} \otimes_{\mathcal{O}} M\right)=L \otimes_{\lambda} M .
$$

Hence $\mu_{*}\left(\varphi \otimes K^{\prime}\right)=\lambda_{*}(\varphi)$.

In the following $\varphi$ is a nondegenerate form over a field $k$.

Scholium 6.3. Let $K \supset k, L \supset k$ be field extensions of $k$, and let $\lambda: K \rightarrow L \cup \infty$ be a place over $k$, i.e., a place extending the trivial place $k \hookrightarrow L$. Assume that $L$ is $\varphi$-conservative. Then Theorem 6.2 tells us that $K$ is $\varphi$-conservative, $\varphi \otimes K$ has $G R$ with respect to $\lambda$, and $\lambda_{*}(\varphi \otimes K)=\varphi \otimes L$.

Let $\varphi \otimes K \cong \varphi_{1} \perp r_{1} \times H$ be the Witt decomposition of $\varphi$. By Theorem 5.12 it follows that $\varphi_{1}$ has GR with respect to $\lambda$, and hence

$$
\varphi \otimes L=\lambda_{*}(\varphi \otimes K)=\lambda_{*}\left(\varphi_{1}\right) \perp r \times H
$$

\{We denote the hyperbolic plane $\left[\begin{array}{ll}0 & 1 \\ 1 & 0\end{array}\right]$ over any field (or ring) by $\left.H.\right\}$

Thus $\operatorname{ind}(\varphi \otimes L) \geq \operatorname{ind}(\varphi \otimes K)$, and, in case of equality,

$$
\operatorname{ker}(\varphi \otimes L)=\lambda_{*}(\operatorname{ker}(\varphi \otimes K))
$$

It now follows that, if $K$ and $L$ are specialization equivalent over $k$, i.e., there exists also a place over $k$ from $L$ to $K$, then

$$
\operatorname{ind}(\varphi \otimes L)=\operatorname{ind}(\varphi \otimes K),
$$

and

$$
\operatorname{ker}(\varphi \otimes L)=\lambda_{*}(\operatorname{ker} \varphi \otimes K)
$$

for any place $\lambda$ from $K$ to $L$ over $k$.

Definition 6.4. We call a field extension $K \supset k$ a generic zero field of $\varphi$, if $K$ is $\varphi$-conservative, and there exists a place from $K$ to $L$ over $\varphi$ for any $\varphi$-conservative field extension $L \supset k$ such that $\varphi \otimes L$ is isotropic.

Notice that $\varphi \otimes L$ is isotropic iff $\operatorname{ind}(\varphi \otimes L) \geq 1$, since $\varphi \otimes L$ is nondegenerate.

Any two generic zero fields of $\varphi$ are specialization equivalent over $k$.

Fortunately generic zero fields of $\varphi$ exist whenever this makes sense.

Theorem 6.5. Assume that $\varphi$ is anisotropic and not quasilinear, $n:=\operatorname{dim} \varphi \geq 2$.

a) The function field $k(\varphi)$ of the affine quadric $\varphi\left(x_{1}, \ldots, x_{n}\right)=0$, i.e., the quotient field of the integral domain

$$
k\left[x_{1}, \ldots, x_{n}\right] /\left(\varphi\left(x_{1}, \ldots, x_{n}\right)\right)
$$

is a generic zero field of $\varphi .\{N . B . k(\varphi)$ is separable over $k$. 
b) More generally the following holds. If $\gamma: k \rightarrow L \cup \infty$ is a place such that $\varphi$ has GR under $\gamma$, and if $\gamma_{*}(\varphi)$ is nondegenerate and isotropic, there exists a place $\lambda: k(\varphi) \rightarrow L \cup \infty$ extending $\gamma$.

We now can build a generic splitting tower $\left(K_{r} \mid 0 \leq r \leq h\right)$ of $\varphi$ in the way well known from the case that char $K \neq 2$ (cf. $[\mathrm{K}],[\mathrm{KS}],[\mathrm{S}], \ldots)$ and from the case that $\operatorname{char} k=2$, but $\varphi$ regular (cf. $[\mathrm{KR}]$ ).

Take $K_{0}=k$, or more generally, let $K_{0}$ be field extension of $k$ such that there exists a place from $K_{0}$ to $k$ over $k$, with corresponding Witt decomposition

$$
\varphi \otimes K_{0} \cong \varphi_{0} \perp i_{0} \times H
$$

(N.B.: $i_{0}=\operatorname{ind}(\varphi)$ ). If $\varphi_{0}$ is quasilinear, we stop.

Otherwise we choose a generic zero field $K_{1} \supset K_{0}$ of $\varphi_{0}$, and then have a Witt decomposition

$$
\varphi_{0} \otimes K_{1} \cong \varphi_{1} \perp i_{1} \times H
$$

etc. We could take $K_{0}=k, K_{1}=k\left(\varphi_{0}\right)$, etc. But for various problems it is useful to allow other generic splitting towers.

We retain the terminology from the generic splitting theory in characteristic $\neq 2$. In particular we call $i_{r}$ the $r$-th higher index of $\varphi$ and $h$ the height of $\varphi$. The form $\varphi_{h}$ is quasilinear.

Precisely as in the characteristic $\neq 2$ case we obtain from the above theorems immediately:

Theorem 6.6. Let $\varphi$ be a non degenerate form over $k$. Let $\left(K_{r} \mid 0 \leq r \leq h\right)$ be a generic splitting tower of $\varphi$ with associated higher indices $i_{r}$ and higher kernel forms $\varphi_{r}$. Let $\gamma: k \rightarrow L \cup \infty$ be a place such that $\varphi$ has GR with respect to $\gamma$ and $\gamma_{*}(\varphi)$ is non degenerate. We choose a place $\lambda: K_{m} \rightarrow L \cup \infty$ extending $\gamma$ such that either $m=r$ as $m<r$, but $\lambda$ cannot be extended to a place from $K_{m+1}$ to $L$. Then $\varphi_{m}$ has GR with respect to $\lambda$. The form $\lambda_{*}(\varphi)$ has the kernel form $\lambda_{*}\left(\varphi_{m}\right)$ and the Witt index $i_{0}+\ldots+i_{m}$.

If $L \supset k$ is a $\varphi$-conservative field extension we may apply the theorem to the trivial place $\gamma: k \hookrightarrow L$ and obtain precise information about the Witt decomposition of $\varphi \otimes L$.

\section{EPILOGUe}

A) Perhaps the most urgent open problem in generic splitting theory is to determine all forms of height 1 . Assume that $\varphi$ is anisotropic and $h(\varphi)=1$. If $\varphi$ is strictly regular, then it turns out that $\varphi$ is, up to a scalar factor, a quadratic Pfister form. (cf. [Spez, §20]). If $Q L(\varphi)$ has dimension 1 then $\varphi$ is, up to scalar factor, a certain "close neighbor" of a quadratic Pfister form (cf. [Spez, §22]), analogous to the pure part of a Pfister form in the case of characteristic $\neq 2$. But, if $\operatorname{dim} Q L(\varphi) \geq 2$ there exist more forms of height 1 than those which are Witt equivalent to close Pfister neighbors. 
B) Let $K$ and $L$ be fields with $\operatorname{char} K=0$, $\operatorname{char} L=2$. Given a place $\lambda: K \rightarrow L \cup \infty$, it deserves interest to "lift" a nondegenerate quadratic form $\psi$ over $L$ to a form $\varphi$ over $K$, i.e., to exhibit a quadratic form $\varphi$ over $K$ with GR with respect to $\lambda$ and $\lambda_{*}(\varphi) \cong \psi$. Then one can hope to deduce properties of $\psi$ from properties of $\varphi$.

In the specialization theory outlined above such a lifting is only possible if $\psi$ is strictly regular. Indeed, since a nondegenerate form $\varphi$ over $K$ as automatically strictly regular, also $\lambda_{*}(\varphi)$ has to be strictly regular.

Fortunately there exists a more general specialization theory than the one explicated in $\S 5$.

Given a place $\lambda: K \rightarrow L \cup \infty$ with valuation ring $\mathcal{O}$, we say that a quadratic space $E=(E, q)$ over $K$ has fair reduction with respect to $\lambda$, if $E$ contains a free $\mathcal{O}$-submodule $M$ with $E=K M$ and $q(M) \subset \mathcal{O}$, such that $\left(M / \mathfrak{m} M, \overline{\left.q\right|_{M}}\right)$ is a quadratic space over $\mathcal{O} / \mathfrak{m}$, while $\left(M,\left.q\right|_{M}\right)$ may be degenerate. One can prove that then

$$
\lambda_{*}(E):=L \otimes_{\bar{\lambda}}(M / \mathfrak{m} M)=L \otimes_{\lambda} M
$$

is still well defined up to isometry by $E$ and $\lambda$. This is the basis of a "fair specialization theory" which parallels our theory in $\S 5$, cf. [Spez, II, §11].

It is now well possible to find for a non degenerate form $\psi$ over $L$ a form $\varphi$ over $K$ with fair reduction with respect to $\lambda$ and $\lambda_{*}(\varphi) \cong \psi$. For fair specializations there also exists a theorem completely analogous to the generic splitting theorem 6.6 above, cf. [Spez, II, §12].

\section{REFERENCES}

[Spez] M.Knebusch, "Spezialisierung von quadratischen und symmetrischen bilinearen Formen" (2007),

http://www-nw.uni-regensburg.de/ .knm22087.mathematik.uniregensburg.de/index.htm (the author's homepage).

[Spez'] Translation of [Spez] by Thomas Unger, in preparation. Chapter I and II available as preprint.

[K] M. Knebusch, "Generic splitting of quadratic forms I", Proc. London Math. Soc. (3) 33 (1976), 65-93.

[KR] M. Knebusch, U. Rehmann, "Generic splitting towers and generic splitting preparation", Contemporary Math. 272 (2000), 173-199.

[KR] M. Knebusch, W. Scharlau, "Algebraic theory of quadratic forms, generic methods and Pfister forms", DMV-Seminar N 1 (Notes taken by Heisook Lee). Birkhauser 1980. 
[S] W. Scharlau, "Quadratic and hermitian forms" (Grundlehren Math. Wiss. 270), Springer 1985.

Universität Regensburg, NWF I - Mathematik, D-93040 Regensburg, Germany

E-mail address: manfred.knebusch@mathematik.uni-regensburg.de 